\title{
Plasma Alarin Level and Its Influencing Factors in Obese Newly Diagnosed Type 2 Diabetes Patients
}

This article was published in the following Dove Press journal:

Diabetes, Metabolic Syndrome and Obesity: Targets and Therapy

\section{Xin Zhou* \\ Mei Luo* \\ Sha Zhou \\ Zhiling Cheng \\ Zhongpei Chen \\ Xiaoxia Yu}

Department of Endocrinology, Chongqing Traditional Chinese Medicine Hospital, Chongqing 400000, People's Republic of China

*These authors contributed equally to this work
Correspondence: Xiaoxia Yu Department of Endocrinology, Chongqing Traditional Chinese Medicine Hospital, Chongqing 400000, People's Republic of China

$\mathrm{Tel} / \mathrm{Fax}+862363842760$

Email yudoctor789@163.com
Objective: To investigate the plasma alarin level in newly diagnosed obese type 2 diabetes mellitus (T2DM) and its correlation with glucose and lipid metabolism and insulin resistance. Methods: From October 2018 to June 2020, 239 newly diagnosed T2DM patients were collected. According to obesity, patients were divided into T2DM obese group $(n=135)$ and T2DM non-obese group $(\mathrm{n}=104)$. Gender, age, body mass index (BMI), blood lipids, blood glucose, glycosylated hemoglobin A1c (HbA1c), fasting insulin (FINS), plasma alarin concentration, homeostasis model assessment for insulin resistance (HOMA-IR), homeostasis model assessment for $\beta$-cell function (HOMA- $\beta$ ) and other clinical data were collected and analyzed. Results: BMI, triacylglycerol (TG), total cholesterol (TC), low-density lipoproteincholesterol (LDL-C), fasting blood glucose (FPG), HbA1c, FINS, plasma alarin levels and HOMA-IR in the control group, T2DM non-obese group and T2DM obese group increased sequentially, and high-density lipoprotein-cholesterol (HDL-L) and HOMA- $\beta$ decreased sequentially $(\mathrm{P}<0.05)$. Correlation analysis results showed that plasma alarin levels in T2DM patients were positively correlated with waistline, BMI, TC, LDL-C, FPG, HbA1c, FINS and HOMA-IR $(\mathrm{P}<0.05)$, and negatively correlated with HDL-C and HOMA- $\beta$ (P $<0.05$ ), and the correlation coefficient of T2DM obese group was significantly higher than that of $\mathrm{T} 2 \mathrm{DM}$ non-obese group $(\mathrm{P}<0.05)$. Multiple linear stepwise regression analysis showed that BMI, FPG, HbA1c, HOMA- $\beta$, and HOMA-IR were independent factors related to plasma alarin levels in T2DM non-obese and T2DM obese patients, and the correlation coefficient of the T2DM obese group was significantly higher than that of the T2DM nonobese group ( $\mathrm{P}<0.05)$.

Conclusion: Plasma alarin levels increase in newly diagnosed T2DM and obese T2DM patients, which are affected by TC, BMI, FPG, HbA1c, HOMA- $\beta$ and HOMA-IR, and may be involved in development of T2DM.

Keywords: type 2 diabetes mellitus, obesity, plasma alarin level, insulin resistance, blood sugar, blood lipids, insulin

\section{Introduction}

Diabetes is a metabolic disease characterized by hyperglycemia due to insulin secretion deficiency or insulin dysfunction. At present, more than $90 \%$ of the diabetes mellitus patients in the world have type 2 diabetes mellitus (T2DM). The incidence of T2DM is increasing year by year, and there is a younger trend. ${ }^{1,2}$ It is estimated that by 2040 , the total global T2DM population will reach 642 million people. Epidemiological surveys show that the main driving factors for the global type 2 diabetes epidemic are rising obesity rates, sedentary lifestyles, high-energy diets and an aging population, among which obesity is the most 
important independent risk factor for T2DM. ${ }^{2-4}$ The occurrence and development of obesity are closely related to the body's food intake and energy expenditure. In the state of excess energy, it can promote the occurrence of obesity, and obesity is closely related to the occurrence and development of T2DM and insulin resistance (IR). ${ }^{4,5}$

Alarin is the newest member of the galanin neuropeptide family. It is a peptide composed of 25 amino acids, which is involved in the body's food intake regulation and energy metabolism. ${ }^{6,7}$ Studies have confirmed that intracerebroventricular injection of alarin in rats can promote the increase in food intake and body weight of rats. ${ }^{8-10}$ Current research suggests that alarin plays an important role in the occurrence and development of diabetes and obesity. ${ }^{11-13}$ However, there are few reports on the relationship between alarin and the glucose and lipid metabolism and insulin resistance of newly diagnosed T2DM with obesity. This article explores the relationship between plasma alarin levels and glucose and lipid metabolism and IR in obese newly diagnosed T2DM patients, and provides reference for the study of the mechanism of alarin in the development of diabetes.

\section{Patients and Methods}

\section{Patient Inclusion and Exclusion Criteria}

From October 2018 to June 2020, 239 newly diagnosed T2DM patients in our hospital were included in the study. The criteria for diagnosis and classification of diabetes mellitus formulated by the World Health Organization in 1999. ${ }^{14}$ Inclusion criteria were as follows: 1) patients with T2DM symptoms for less than 1 year; 2) patients were not treated with any hypoglycemic and lipid-lowering drugs; $3)$ patients were 18-65 years old. Exclusion criteria were as follows: 1) excluding patients with type 1 diabetes mellitus, gestational diabetes mellitus and other special types of diabetes, acute and chronic complications of diabetes mellitus; 2) patients with secondary obesity caused by other reasons, such as hypothyroidism; 3) patients with severe cardiovascular and cerebrovascular diseases and liver and kidney dysfunction; 4) pregnant and lactating women; 5) Patients with age $<18$ years or $>65$ years; 6) patients with mental illness; 7) patients who take glucocorticoids and receive diabetes-related treatment affect blood glucose monitoring results; 8) patients with stress such as trauma and surgery. Patients were divided into two groups according to obesity: T2DM obese group $(\mathrm{n}=135)$ and T2DM non-obese group $(\mathrm{n}=104)$. The diagnostic criteria for obesity refer to research reports and the constitution of Asian populations. Those with a body mass index of $18.5 \mathrm{~kg} / \mathrm{m}^{2} \leq$ body mass index $<24 \mathrm{~kg} / \mathrm{m}^{2}$ are considered non-obese, and those with a body mass index $\geq 25 \mathrm{~kg} / \mathrm{m}^{2}$ are considered obese. ${ }^{15-17}$ Select 85 healthy non-obese people who underwent health checkups in our hospital during the same period as the normal control group. Inclusion criteria were as follows: 1) fasting blood glucose and postprandial blood glucose were normal; 2) never took drugs that affect blood sugar and blood lipids; 3) no stress state, normal liver and kidney function. All procedures performed in studies involving human participants were in accordance with the ethical standards of the institutional and/or national research committee and with the 1964 Helsinki Declaration and its later amendments or comparable ethical standards. This study was reviewed and approved by the Ethics Committee of Chongqing Hospital of Traditional Chinese Medicine. The subjects gave informed consent to the study, and all subjects signed an informed consent form.

\section{Clinical Data Collection}

The age, gender, BMI, waist circumference, hip circumference, blood sugar, blood lipids and other clinical data of the research subjects were collected and analyzed. The subjects were fasted for 8-10 hours, and blood was drawn from the cubital vein on an empty stomach the next morning for testing. Enzyme method was used to detect FPG, TC, TG, LDL-C, HDL-C through the AU5811 automatic biochemical analyzer produced by Beckman in the United States. Detect HbA1c by highpressure liquid chromatography (provided by ARKRAY, model: HA-8180). Use an automatic electrochemiluminescence detector (Abbott, USA, Model: 05121LP30) to measure FINS, and calculate HOMA-IR index (HOMA-IR $=\mathrm{FPG} \times \mathrm{FINS} / 22.5)$ and HOMA- $\beta$ index [HOMA$\beta=$ FINS $\times 20 /($ FPG-3.5)]. Plasma alarin was detected by enzyme-linked immunosorbent assay (ELISA), and the ELISA Kit were purchased from Becton, Dickinson and Company in the United States, and detected by THERMO FISHER Multiskan FC microplate reader.

\section{Statistical Analysis}

SPSS 22.0 software was used for statistical analysis. Normal distribution of the data was tested using the Kolmogorov-Smirnov test. The variables of non-normal distribution were skewed and logarithmically transformed to obtain a normal distribution. Continuous variables were 
presented as mean $\pm \mathrm{SD}$. Comparisons between groups were performed using ANOVA, an unpaired $t$-test or a paired $t$-test. Simple and multiple linear regression analyses were used to assess the association between circulating alarin levels and the other biomarkers. The difference was statistically significant with $\mathrm{P}<0.05$.

\section{Result}

\section{Comparison of Plasma Alarin Levels,} Glucose and Lipid Metabolism and Insulin Resistance Indexes Among the Three

\section{Groups}

As shown in Table 1, there was no difference in age and gender among the three groups of patients $(\mathrm{P}>0.05)$. Compared with the control group, the T2DM non-obese group and the T2DM obese group had significant increases in waistline, waist-to-hip ratio, BMI, TG, TC, LDL-C, FPG, $\mathrm{HbAlc}$, FINS, plasma alarin concentration and HOMA-IR, while HDL-L and HOMA- $\beta$ directions are significantly reduced $(\mathrm{P}<0.05)$. Compared with the T2DM non-obese group, the T2DM obese group had significant increases in waistline $(\mathrm{P}<0.05)$, waist-to-hip ratio $(\mathrm{P}<0.05)$, BMI (31.01 \pm 1.43 vs23.08 $\pm 1.31, \mathrm{P}<0.05)$, TG $(\mathrm{P}<0.05)$, TC $(5.86 \pm 0.38$ vs $5.13 \pm 0.31, \mathrm{P}<0.05)$, LDL-C $(\mathrm{P}<0.05)$, FPG $(13.61 \pm 1.02$ vs $8.77 \pm 0.86, \mathrm{P}<0.05), \mathrm{HbA1c}(14.64 \pm 1.79$ vs $9.16 \pm 1.36$, $\mathrm{P}<0.05)$, FINS $(\mathrm{P}<0.05)$, plasma alarin concentration $(1.03$ \pm 0.34 VS $0.55 \pm 0.22, \mathrm{P}<0.05)$, and HOMA-IR index $(6.86$ \pm 1.47 VS $4.04 \pm 0.57, \mathrm{P}<0.05)$, while in HDL-L $(1.01 \pm 0.25$ VS $1.32 \pm 0.23, \mathrm{P}<0.05)$ and HOMA- $\beta(23.53 \pm 5.07$ VS $39.47 \pm 6.24, \mathrm{P}<0.05)$ are significantly reduced.

\section{Linear Regression Analysis of the Relationship Between Plasma Alarin Levels, Glucose and Lipid Metabolism and Insulin Resistance Indexes in Three \\ Groups}

As shown in Table 2, the plasma alarin level in the control group was not significantly correlated with Waistline, waist-to-hip ratio, BMI, TG, TC, LDL-C, FPG, HbAlc, FINS, HOMA-IR, HDL-C and HOMA- $\beta(\mathrm{P}>0.05)$. In the T2DM non-obese group, plasma alarin levels were not significantly correlated with Waistline, waist-to-hip ratio and TG $(\mathrm{P}>0.05)$, but positively correlated with TC $(\mathrm{r}=0.206, \mathrm{P}=0.005)$, BMI $(\mathrm{r}=0.304, \mathrm{P}=0.007)$, LDL-C $(\mathrm{r}=0.234, \quad \mathrm{P}=0.011)$, FPG $(\mathrm{r}=0.394, \mathrm{P}<0.001), \mathrm{HbA} 1 \mathrm{c}$ $(\mathrm{r}=0.318, \mathrm{P}<0.001)$, FINS $(\mathrm{r}=0.124, \mathrm{P}=0.021)$ and HOMAIR $(\mathrm{r}=0.418, \mathrm{P}<0.001)$, and negatively correlated with HDL-C $(r=-0.273, P=0.017)$ and HOMA- $\beta(r=0.347$, $\mathrm{P}<0.001)$. Plasma alarin levels in obese patients with T2DM were positively correlated with Waistline $(\mathrm{r}=0.244, \quad \mathrm{P}=0.023), \quad$ BMI $\quad(\mathrm{r}=0.523, \quad \mathrm{P}<0.001), \quad \mathrm{TC}$ $(\mathrm{r}=0.352, \quad \mathrm{P}<0.001), \quad$ LDL-C $(\mathrm{r}=0.326, \mathrm{P}=0.002)$, FPG $(\mathrm{r}=0.578, \mathrm{P}<0.001), \mathrm{HbA1c}(\mathrm{r}=0.481, \mathrm{P}<0.001)$, FINS $(\mathrm{r}=0.245, \mathrm{p}=0.017)$ and HOMA-IR $(\mathrm{r}=0.674, \mathrm{P}<0.001)$,

Table I Comparison of Plasma Alarin Levels, Glucose and Lipid Metabolism and Insulin Resistance Indicators in the Three Groups

\begin{tabular}{|c|c|c|c|}
\hline Parameters & Control Group $(n=85)$ & T2DM Non-Obese Group $(n=104)$ & T2DM Obesity Group $(n=135)$ \\
\hline Age (year) & $41.54 \pm 7.56$ & $42.14 \pm 8.02$ & $43.32 \pm 6.12$ \\
\hline Gender (Male/Female) & $35 / 30$ & $58 / 46$ & $72 / 63$ \\
\hline Waistline $(\mathrm{cm})$ & $72.21 \pm 9.01$ & $81.39 \pm 10.14$ & $112.02 \pm 10.62^{*}, \#$ \\
\hline Waist-to-hip ratio & $0.78 \pm 0.07$ & $0.8 I \pm 0.06$ & $0.96 \pm 0.05^{*}, \#$ \\
\hline $\mathrm{BMI}\left(\mathrm{kg} / \mathrm{m}^{2}\right)$ & $20.47 \pm 1.12$ & $23.08 \pm 1.31^{*}$ & $31.01 \pm 1.43^{*, \#}$ \\
\hline $\mathrm{TG}(\mathrm{mmol} / \mathrm{L})$ & $1.06 \pm 0.14$ & $2.01 \pm 0.22 *$ & $2.57 \pm 0.29^{*}, \#$ \\
\hline $\mathrm{TC}(\mathrm{mmol} / \mathrm{L})$ & $4.03 \pm 0.27$ & $5.13 \pm 0.31 *$ & $5.86 \pm 0.38^{*}, \#$ \\
\hline HDL-C (mmol/L) & $1.63 \pm 0.18$ & $1.32 \pm 0.23 *$ & $1.01 \pm 0.25^{*}, \#$ \\
\hline LDL-C (mmol/L) & $2.65 \pm 0.28$ & $3.45 \pm 0.34 *$ & $4.01 \pm 0.41^{*, \#}$ \\
\hline FPG (mmol/L) & $4.79 \pm 0.37$ & $8.77 \pm 0.86 *$ & $\mid 3.61 \pm 1.02^{*}, \#$ \\
\hline HbAlc (\%) & $5.0 I \pm 0.4 I$ & $9.16 \pm 1.36 *$ & $14.64 \pm 1.79^{*}, \#$ \\
\hline FINS (mU/L) & $5.44 \pm 2.01$ & $9.26 \pm 3.47 *$ & $12.84 \pm 3.67^{*}, \#$ \\
\hline Alarin $(\mu g / L)$ & $0.21 \pm 0.12$ & $0.55 \pm 0.22 *$ & $1.03 \pm 0.34^{*}, \#$ \\
\hline HOMA-IR & $1.12 \pm 0.31$ & $4.04 \pm 0.57 *$ & $6.86 \pm 1.47^{*, \#}$ \\
\hline HOMA $\square \beta$ & $79.84 \pm 4.54$ & $39.47 \pm 6.24^{*}$ & $23.53 \pm 5.07^{*}, \#$ \\
\hline
\end{tabular}

Notes: *Indicates compared with the control group, $\mathrm{P}<0.05$; ${ }^{\#}$ Indicates compared with the T2DM non-obese group, $\mathrm{P}<0.05$.

Abbreviations: BMI, body mass index; TG, triacylglycerol; TC, total cholesterol; LDL-C, low-density lipoprotein-cholesterol; HDL-C, high-density lipoprotein-cholesterol; FPG, fasting blood glucose; FINS, fasting insulin; HbAIc, glycosylated hemoglobin Alc; HOMA-IR, homeostasis model assessment for insulin resistance; HOMA- $\beta$, homeostasis model assessment for $\beta$-cell function. 
Table 2 Linear Regression Analysis of the Relationship Between Plasma Alarin Levels, Glucose and Lipid Metabolism and Insulin Resistance Indexes in Three Groups

\begin{tabular}{|c|c|c|c|c|c|c|}
\hline \multirow[t]{2}{*}{ Parameters } & \multicolumn{2}{|c|}{ Control Group $(n=85)$} & \multicolumn{2}{|c|}{ T2DM Non-Obese Group $(n=104)$} & \multicolumn{2}{|c|}{ T2DM Obesity Group $(n=135)$} \\
\hline & $r$ value & Corrected $\mathrm{p}$ value & $r$ value & Corrected $\mathrm{p}$ value & $r$ value & Corrected $\mathrm{p}$ value \\
\hline Waistline $(\mathrm{cm})$ & 0.034 & 1.000 & 0.157 & 1.000 & 0.244 & $0.023 *$ \\
\hline Waist-to-hip ratio & 0.015 & 1.000 & 0.132 & 1.000 & 0.147 & 1.000 \\
\hline BMI $\left(\mathrm{kg} / \mathrm{m}^{2}\right)$ & 0.171 & 1.000 & 0.304 & $0.007^{*}$ & 0.523 & $<0.001 *$ \\
\hline TG (mmol/L) & 0.024 & 1.000 & 0.157 & 1.000 & 0.226 & 1.000 \\
\hline $\mathrm{TC}(\mathrm{mmol} / \mathrm{L})$ & 0.054 & 1.000 & 0.206 & $0.005^{*}$ & 0.352 & $<0.00$ I* \\
\hline HDL-C (mmol/L) & 0.045 & 1.000 & -0.273 & $0.017^{*}$ & -0.341 & $0.013^{*}$ \\
\hline LDL-C (mmol/L) & 0.071 & 1.000 & 0.234 & $0.011 *$ & 0.326 & $0.002 *$ \\
\hline FPG (mmol/L) & 0.094 & 1.000 & 0.394 & $<0.001 *$ & 0.578 & $<0.001 *$ \\
\hline $\mathrm{HbAlc}(\%)$ & 0.104 & 1.000 & 0.318 & $<0.001 *$ & 0.481 & $<0.00 I^{*}$ \\
\hline FINS (mU/L) & 0.085 & 1.000 & 0.124 & $0.021^{*}$ & 0.245 & $0.017^{*}$ \\
\hline HOMA-IR & 0.091 & 1.000 & 0.418 & $<0.001 *$ & 0.674 & $<0.001 *$ \\
\hline HOMA- $\beta$ & 0.224 & 1.000 & -0.347 & $<0.001 *$ & -0.557 & $<0.001 *$ \\
\hline
\end{tabular}

Note: *Represents $\mathrm{P}<0.05$ after correction.

Abbreviations: BMI, body mass index; TG, triacylglycerol; TC, total cholesterol; LDL-C, low-density lipoprotein-cholesterol; HDL-C, high-density lipoprotein-cholesterol; FPG, fasting blood glucose; FINS, fasting insulin; HbAlc, glycosylated hemoglobin Alc; HOMA-IR, homeostasis model assessment for insulin resistance; HOMA- $\beta$, homeostasis model assessment for $\beta$-cell function.

and negatively correlated with HDL-C $(\mathrm{r}=-0.341$, $\mathrm{p}=0.013)$ and HOMA- $\beta(\mathrm{r}=-0.557, \mathrm{P}<0.001)$, and the correlation coefficient was significantly higher than that of T2DM non-obesity group.

\section{Multiple Linear Regression Analysis of Plasma Alarin Levels, Glucose and Lipid Metabolism and Insulin Resistance Indexes in T2DM Obese Group and T2DM Non-Obese Group}

As shown in Table 3, BMI, FPG, HbA1c, HOMA- $\beta$, and HOMA-IR are independent factors related to plasma alarin levels in non-obese T2DM and obese T2DM patients $(\mathrm{P}<0.05)$. Among them, TC, BMI, FPG, HbA1c and HOMA-IR are positively correlated, HOMA-IR -B negative correlation $(\mathrm{P}<0.05)$. Compared with non-obese T2DM, the correlation coefficients of $\mathrm{TC}(\mathrm{P}<0.001)$, BMI $\quad(\mathrm{P}<0.001)$, FPG $(\mathrm{P}<0.001)$, HbA1c $\quad(\mathrm{P}=0.005)$, HOMA- $\beta \quad(p=0.002)$ and HOMA-IR $(P<0.001)$ in the obese T2DM group were higher, which indicated that T2DM with obesity had a more significant effect on plasma alarin levels.

\section{Discussion}

Obesity, as a disorder of energy metabolism, has become a worldwide health problem. ${ }^{18,19}$ Many studies have shown that obesity is closely related to the occurrence and development of T2DM, hypertension, hyperlipidemia, coronary heart disease and other diseases. ${ }^{18-20}$ When the body has excess energy, the energy will be stored in the body in the form of fat, leading to the proliferation and hypertrophy of fat cells, causing obesity. Excessively enlarged fat cells can produce a variety of cytokines, which can interfere with glucose and lipid metabolism, reduce insulin sensitivity and lead to insulin resistance. ${ }^{21,22}$ Studies have shown that obesity, especially central obesity, is the main risk factor for IR, and the occurrence of insulin resistance (IR) is closely related to the degree of obesity. ${ }^{23,24}$ Obesity and diabetic patients often have glucose and lipid metabolism disorders and IR, ${ }^{21-24}$ As the most basic pathological mechanism of T2DM, IR continues throughout the entire course of T2DM. In this study, we found that compared with the control group, BMI, TG, TC, LDL-C, FPG, HbA1c, FINS and HOMA-IR were significantly increased in the T2DM non-obese group and T2DM obese group, while HDL-L and HOMA- $\beta$ were significantly reduced, and this trend was more obvious in the T2DM obese group. This result suggests that the disorder of glucose and lipid metabolism in obese T2DM patients is more serious than that in nonobese T2DM. Abnormal glucose and lipid metabolism aggravates the occurrence and development of IR and pancreatic dysfunction in obese T2DM patients.

Studies have shown that the galanin neuropeptide family is involved in the regulation of appetite, insulin 
Table 3 Multiple Linear Regression Analysis of Plasma Alarin Levels, Glucose and Lipid Metabolism and Insulin Resistance Indexes in T2DM Obese Group and T2DM Non-Obese Group

\begin{tabular}{|c|c|c|c|c|c|c|}
\hline \multirow[t]{2}{*}{ Parameters } & \multicolumn{3}{|c|}{ Control Group $(n=85)$} & \multicolumn{3}{|c|}{ T2DM Obesity Group $(n=135)$} \\
\hline & $\beta$ value & $95 \% \mathrm{Cl}$ & Corrected $\mathbf{P}$ value & $\beta$ value & $95 \% \mathrm{Cl}$ & Corrected $\mathbf{P}$ value \\
\hline Waistline $(\mathrm{cm})$ & - & - & - & 0.347 & $0.094-0.247$ & 0.464 \\
\hline BMI $\left(\mathrm{kg} / \mathrm{m}^{2}\right)$ & 1.321 & $1.074-4.145$ & $<0.00 I^{*}$ & 2.124 & $1.241-6.848$ & $<0.00 I^{*}$ \\
\hline TG (mmol/L) & 0.431 & $0.173-1.319$ & $0.007 *$ & $0.7 \mid 4$ & $0.249-1.758$ & $<0.001 *$ \\
\hline $\mathrm{TC}(\mathrm{mmol} / \mathrm{L})$ & - & - & - & 0.345 & $0.124-0.542$ & 0.392 \\
\hline HDL-C (mmol/L) & -0.481 & $-0.282-1.374$ & 0.304 & 0.759 & $-0.342-1.814$ & 0.221 \\
\hline LDL-C (mmol/L) & $0.65 I$ & $0.235-1.982$ & 0.534 & 1.245 & $0.67 I-2.525$ & 0.402 \\
\hline FPG $(\mathrm{mmol} / \mathrm{L})$ & $0.74 I$ & $0.376-|.57|$ & $0.006 *$ & $\mathrm{I} .46 \mathrm{I}$ & $0.784-3.271$ & $<0.001 *$ \\
\hline HbAlc (\%) & 0.914 & $0.347-2.387$ & $0.010 *$ & $\mathrm{I} .734$ & $0.984-4.756$ & $0.005^{*}$ \\
\hline FINS (mU/L) & 0.324 & $0.114-0.724$ & 0.304 & 0.543 & $0.106-1.013$ & 0.247 \\
\hline HOMA-IR & 1.141 & $0.634-3.578$ & $<0.001 *$ & 1.946 & $1.340-5.378$ & $<0.001 *$ \\
\hline НОМА- $\beta$ & 0.571 & $0.134-1.314$ & $0.008 *$ & 0.861 & $0.270-2.7865$ & $0.002 *$ \\
\hline
\end{tabular}

Note: *Represents $\mathrm{P}<0.05$ after correction.

Abbreviations: BMI, body mass index; TG, triacylglycerol; TC, total cholesterol; LDL-C, low-density lipoprotein-cholesterol; HDL-C, high-density lipoprotein-cholesterol; FPG, fasting blood glucose; FINS, fasting insulin; HbAlc, glycosylated hemoglobin Alc; HOMA-IR, homeostasis model assessment for insulin resistance; HOMA- $\beta$, homeostasis model assessment for $\beta$-cell function.

resistance, obesity, hypertension and metabolism., ${ }^{4,5}$ Alarin is the newly discovered galanin neuropeptide family member and was first discovered in the ganglion cells of neuroblastoma. Follow-up studies have confirmed that Alarin also exists in the perivascular area of the skin, locus coeruleus, arcuate nucleus, olfactory bulb, amygdala, and so on. At present, further studies have shown that the administration of alarin stimulation to the cerebral ventricle and periphery of rats can reduce the blood glucose level of T2DM rats, promote the uptake of skeletal muscle glucose, and improve IR in rats. $^{25-27}$ These studies suggest that alarin is a regulatory factor related to the body's diet and energy balance, which is closely related to the occurrence and development of diabetes mellitus. For example, leptin, which can inhibit appetite, can inhibit insulin secretion. When insulin secretion increases, it can promote leptin secretion, thus regulating glucose and lipid metabolism and participating in the occurrence and development of diabetes and obesity. ${ }^{28,29}$

As a regulator of diet and energy balance, there are few reports on the relationship between alarin and IR, glucose and lipid metabolism in diabetic patients. Hu et al found that serum alarin level was significantly increased in patients with impaired glucose tolerance (IGT) and T2DM, indicating that circulating alarin gradually increased from pre-diabetic state to diabetic stage. ${ }^{11}$ In this study, we found that alarin levels were elevated in both the T2DM non-obese group and the T2DM obese group. But the T2DM obesity group was significantly higher than the T2DM non-obese group, which indicated that the T2DM with obesity patients had a more significant effect on plasma alarin levels. In the linear correlation analysis, we found that the plasma alarin level was positively correlated with waistline, BMI, TG, TC, LDL-C, FPG, HbA1c, Fins, HOMA-IR, and negatively correlated with HDL-C and HOMA- $\beta$, and the correlation coefficient was significantly higher than that of T2DM non-obese group. In the further multiple regression analysis, we found that TC, BMI, FPG, HbA1c, HOMA- $\beta$ and HOMAIR were the independent factors influencing the plasma alarin level in diabetic patients. In addition, compared with non-obese T2DM, the correlation of TC, BMI, FPG, HbA1c, HOMA - $\beta$ and HOMA-IR was higher in the obese T2DM group. These results suggest that alarin may be related to the occurrence and development of T2DM, and the increase of alarin level may be an early defense response to obesity, IR and glucose and lipid metabolism disorders. Of course, we still can not exclude that high level of alarin is a risk factor for the occurrence and development of diabetes, so more in-depth prospective studies are needed to clarify the relationship between them.

Our research also has some limitations. First of all, this study is a cross-sectional study, so we cannot infer the causal relationship between circulating alarin, obesity and T2DM, and further prospective studies are needed to illustrate the exact relationship between them. Second, this study is a small sample, single-center study, and the results 
of the study have limitations. Large samples and multicenter studies further are needed to confirm the results of this study. Third, due to genetic, population, diet and other differences, the results of this study have inherent errors and biases. Fourth, this study has inherent limitations and risks of bias in research design.

\section{Conclusion}

In conclusion, the plasma alarin level was increased in newly diagnosed T2DM and obese T2DM patients and which was correlated with independent risk factors, such as TC, BMI, FPG, HbA1c, HOMA- $\beta$ and HOMA-IR.

\section{Acknowledgments}

Current work was supported by Project of Chongqing Science and health (Fund No. ZY201702030).

\section{Disclosure}

The authors declare that they have no conflicts of interest for this work.

\section{References}

1. Magliano DJ, Sacre JW, Harding JL, et al. Young-onset type 2 diabetes mellitus - implications for morbidity and mortality. Nat Rev Endocrinol. 2020;16(6):321-331. doi:10.1038/s41574-0200334-Z

2. Zheng Y, Ley SH, Hu FB. Global aetiology and epidemiology of type 2 diabetes mellitus and its complications. Nat Rev Endocrinol. 2018;14(2):88-98. doi:10.1038/nrendo.2017.151

3. Zimmet P, George Alberti K, Magliano DJ, et al. Diabetes mellitus statistics on prevalence and mortality: facts and fallacies. Nat Rev Endocrinol. 2016;12(10):616-622. doi:10.1038/nrendo.2016.105

4. Lorber D. Importance of cardiovascular disease risk management in patients with type 2 diabetes mellitus. Diabetes Metab Syndr Obes. 2014;7:169-183. doi:10.2147/DMSO.S61438

5. Yang Q, Vijayakumar A, Kahn BB. Metabolites as regulators of insulin sensitivity and metabolism. Nat Rev Mol Cell Biol. 2018;19 (10):654-672.

6. Fang P, Yu M, Shi M, et al. Galanin peptide family as a modulating target for contribution to metabolic syndrome. Gen Comp Endocrinol. 2012;179(1):115-120. doi:10.1016/j.ygcen.2012.07.029

7. Lang R, Gundlach AL, Kofler B. The galanin peptide family: receptor pharmacology, pleiotropic biological actions, and implications in health and disease. Pharmacol Ther. 2007;115(2):177-207. doi:10.1016/j.pharmthera.2007.05.009

8. Santic R, Fenninger K, Graf K, et al. Gangliocytes in neuroblastic tumors express alarin, a novel peptide derived by differential splicing of the galanin-like peptide gene. J Mol Neurosci. 2006;29 (2):145-152. doi:10.1385/JMN:29:2:145

9. Van Der Kolk N, Madison FN, Mohr M, et al. Alarin stimulates food intake in male rats and $\mathrm{LH}$ secretion in castrated male rats. Neuropeptides. 2010;44(4):333-340. doi:10.1016/j.npep.2010.04.001

10. Boughton CK, Patterson M, Bewick GA, et al. Alarin stimulates food intake and gonadotrophin release in male rats. $\mathrm{Br} J$ Pharmacol. 2010;161(3):601-613. doi:10.1111/j.1476-5381.2010.00893.x
11. Hu W, Fan X, Zhou B, et al. Circulating alarin concentrations are high in patients with type 2 diabetes and increased by glucagon-like peptide-1 receptor agonist treatment: an consort-compliant study. Medicine (Baltimore). 2019;98(28):e16428. doi:10.1097/ MD.0000000000016428

12. Mikó A, Balla P, Tenk J, et al. Thermoregulatory effect of alarin, a new member of the galanin peptide family. Temperature (Austin). 2014;1(1):51-56. doi:10.4161/temp.29790

13. Li MQ, Li JY, Xie L. Level of circulating alarin in obese children and its association with insulin resistance. Zhongguo Dang Dai Er Ke Za Zhi. 2019;21(10):983-986.

14. Botas P, Delgado E, Castaño G, et al. Comparison of the diagnostic criteria for diabetes mellitus, WHO-1985, ADA-1997 and WHO-1999 in the adult population of Asturias (Spain). Diabet Med. 2003;20(11):904-908. doi:10.1046/j.1464-5491.2003.01006.x

15. Kanazawa M, Yoshiike N, Osaka T, et al. Criteria and classification of obesity in Japan and Asia-Oceania. World Rev Nutr Diet. 2005;94:1-12. doi:10.1159/000088200

16. De Lorenzo A, Soldati L, Sarlo F, et al. New obesity classification criteria as a tool for bariatric surgery indication. World J Gastroenterol. 2016;22(2):681-703. doi:10.3748/wjg.v22.i2.681

17. Zhang H, Xu H, Song F, et al. Relation of socioeconomic status to overweight and obesity: a large population-based study of Chinese adults. Ann Hum Biol. 2017;44(6):495-501. doi:10.1080/ 03014460.2017.1328072

18. Conway B, Rene A. Obesity as a disease: no lightweight matter. Obes Rev. 2004;5(3):145-151. doi:10.1111/j.1467-789X.2004.00144.x

19. Broughton DE, Moley KH. Obesity and female infertility: potential mediators of obesity's impact. Fertil Steril. 2017;107(4):840-847. doi:10.1016/j.fertnstert.2017.01.017

20. Seravalle G, Grassi G. Obesity and hypertension. Pharmacol Res. 2017;122:1-7. doi:10.1016/j.phrs.2017.05.013

21. Yazicı D, Sezer H. Insulin resistance, obesity and lipotoxicity. $A d v$ Exp Med Biol. 2017;960:277-304.

22. Kahn SE, Hull RL, Utzschneider KM. Mechanisms linking obesity to insulin resistance and type 2 diabetes. Nature. 2006;444 (7121):840-846. doi:10.1038/nature05482

23. Esser N, Legrand-Poels S, Piette J, et al. Inflammation as a link between obesity, metabolic syndrome and type 2 diabetes. Diabetes Res Clin Pract. 2014;105(2):141-150. doi:10.1016/j. diabres.2014.04.006

24. Riobó Serván P. Obesity and diabetes. Nutr Hosp. 2013;28 Suppl 5 (Suppl 5):138-143. doi:10.3305/nh.2013.28.sup5.6929

25. Zhang $\mathrm{Z}, \mathrm{Wu} \mathrm{Y}$, Sheng $\mathrm{S}$, et al. Intracerebroventricular injection of alarin increased glucose uptake in skeletal muscle of diabetic rats. PLoS One. 2015;10(10):e0139327. doi:10.1371/journal. pone. 0139327

26. Guo L, Fang P, Yu M, et al. Central alarin ameliorated insulin resistance of adipocytes in type 2 diabetic rats. $J$ Endocrinol. 2014;223(3):217-225. doi:10.1530/JOE-14-0102

27. Mikó A, Füredi N, Tenk J, et al. Acute central effects of alarin on the regulation on energy homeostasis. Neuropeptides. 2017;64:117-122. doi:10.1016/j.npep.2016.09.001

28. Katsiki N, Mikhailidis DP, Banach M. Leptin, cardiovascular diseases and type 2 diabetes mellitus. Acta Pharmacol Sin. 2018;39 (7):1176-1188.

29. Farr OM, Gavrieli A, Mantzoros CS. Leptin applications in 2015: what have we learned about leptin and obesity? Curr Opin Endocrinol Diabetes Obes. 2015;22(5):353-359. doi:10.1097/ MED.0000000000000184 


\section{Publish your work in this journal}

Diabetes, Metabolic Syndrome and Obesity: Targets and Therapy is an international, peer-reviewed open-access journal committed to the rapid publication of the latest laboratory and clinical findings in the fields of diabetes, metabolic syndrome and obesity research. Original research, review, case reports, hypothesis formation, expert opinion and commentaries are all considered for publication. The manuscript management system is completely online and includes a very quick and fair peer-review system, which is all easy to use. Visit http://www.dovepress.com/testimonials.php to read real quotes from published authors.

Submit your manuscript here: https://www.dovepress.com/diabetes-metabolic-syndrome-and-obesity-targets-and-therapy-journal 